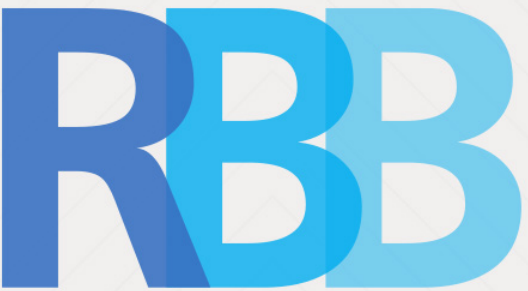

Revista Brasileira de Bioética

Mônica Cristina Sampaio Majewski

Programa de Pós-Graduação em Bioética, PUCPR, Curitiba, PR, Brasil

monica.majewski@pucpr.br

Thiago Rocha da Cunha

Programa de Pós-Graduação em Bioética, PUCPR, Curitiba, PR, Brasil

rocha.thiago@pucpr.pr

\section{Responsabilidade social do farmacêutico à luz das experiências de pacientes: um estudo bioético}

\section{Pharmacist's social responsibility in view of the experiences of patients: a bioethical study}

Resumo: O acompanhamento farmacêutico é um dos serviços clínicos inerentes da profissão farmacêutica consolidado recentemente. Esta pesquisa teve como objetivo analisar a responsabilidade social do farmacêutico a partir da experiência de pacientes atendidos em farmácia comunitária, identificando as experiências com o atendimento farmacêutico ao longo de sua vida adulta. Fez-se um estudo descritivo, exploratório, do tipo qualitativo a partir dos relatos de 15 usuários de uma farmácia universitária do município de Curitiba. Para tanto, foram utilizados dois instrumentos: um Protocolo de Registro de Atendimento Farmacêutico e um roteiro semiestruturado para condução das entrevistas. As experiências relatadas apresentaram um farmacêutico ativo na sociedade, demonstrando a percepção quanto à qualidade geral de serviços farmacêuticos. Porém, ainda há questões importantes a serem considerados na atuação socialmente responsável destes profissionais, principalmente no que se referem aos serviços clínicos, como orientação e acompanhamento farmacoterapêutico, que busca otimização dos resultados terapêuticos, desenvolvendo hábitos de saúde, aumentando a qualidade de vida de toda a população.

Palavras-chave: Serviços comunitários de farmácia, bioética, atenção farmacêutica.

Abstract: Pharmaceutical monitoring, also known as Pharmaceutical Care, is one of the inherent clinical services of the pharmaceutical profession. The present research had as objective to analyze the social responsibility of the pharmacist from the experience of patients attended in community pharmacy, identifying the perception and experiences of these patients with pharmaceutical care throughout their adult life. A descriptive, exploratory, qualitative study was made based on the experiences reports of users of a University Pharmacy in the city of Curitiba. For this, two instruments were used. First, a Pharmaceutical Service Registration Protocol was developed, which categorizes the type of care performed and indicates possible pharmaceutical interventions, followed by a semi-structured script that led to interviews with selected patients. The experiences reported in this research presented a present and active pharmacist in society, demonstrating the progress made in recent years in the quality of pharmaceutical services. However, there are still important issues to be considered in the socially responsible performance of these professionals, especially regarding clinical services, such as orientation and pharmacotherapeutic monitoring, which seeks to optimize therapeutic results, developing health habits, increasing the quality of life of the population.

Keywords: Community pharmacy services, bioethics, pharmaceutical care. 


\section{Introdução}

O acompanhamento farmacêutico, conhecido também como Atenção Farmacêutica, é um dos serviços clínicos inerentes da profissão farmacêutica que surgiu por volta dos anos 1990, nos Estados Unidos. Inicialmente, foi denominado de Farmácia Clínica e era exercida apenas no âmbito hospitalar. Foi então que os autores Hepler e Strand apresentaram uma nova proposta de modelo de prática profissional voltado para as necessidades dos pacientes fora da realidade hospitalar e a definiram como Pharmaceutical Care (Molina, Finkler, 2016). Tal exercício é organizado para resolver problemas sociais de morbidade e mortalidade relacionadas a medicamentos. $\mathrm{Pa}$ cientes que requerem tratamento farmacológico precisam da garantia que tal tratamento será adequado, efetivo e seguro (Cipolle, Strand, Morley, 2006).

Atualmente no Brasil, a prática farmacêutica comunitária, sobretudo em estabelecimentos comerciais privados, é a principal área de trabalho do farmacêutico. A expressão "farmácia comunitária" diz respeito aos estabelecimentos farmacêuticos, que atendem a comunidade, não sendo estabelecimentos hospitalares e ambulatoriais. A necessidade principal refere-se em produzir e disponibilizar medicamentos que atendam padrões de qualidade predefinidos para a população. Tais medicamentos devem ser devidamente prescritos ou indicados, estar acessíveis a população e devem ser corretamente dispensados, baseando-se nas boas práticas de farmácia (Correr, Melchiors, Otuki, 2013).

Os medicamentos são importantes recursos terapêuticos utilizados para tratamento e prevenção de doenças. Tais doenças podem ser classificadas de acordo com o tempo de duração, como agudas ou crônicas. Doenças agudas são enfermidades de curto período, por exemplo, resfriados, dores de garganta e diarreia e as doenças crônicas são de longo período, citando como exemplo Diabetes Mellitus e Hipertensão Arterial Sistêmica. No Brasil, o aumento população idosa resultou em uma maior prevalência de doenças crônicas. Indivíduos acima de 65 anos representavam $4,8 \%$ da população do país, ao passo que em 2010 chegou a 7,4\%. Como resultado, há um crescimento de procura por serviços de saúde e consequentemente por medicamentos (Vianal et al., 2015).

Os farmacêuticos sendo especialistas em medicamentos, são qualificados a resolver problemas relacionados a farmacoterapia, promovendo o uso racional e garantindo efetividade e segurança. A Organização Mundial de Saúde (OMS) preconiza que, para o uso racional de medicamentos, é preciso consolidar a necessidade 
do uso do medicamento. Em seguida, que se prescreva o medicamento adequado, seguindo preceitos de eficácia e segurança comprovadas e na forma farmacêutica, doses e período de duração do tratamento apropriados, o medicamento deve estar disponível, a preço acessível, e que atenda aos padrões de qualidade exigidos e deve ser dispensado com as orientações necessárias para que se cumpra a terapia medicamentosa, da melhor maneira possível (WHO, 1987).

Com o propósito de compreender e refletir eticamente sobre a função do farmacêutico perante a sociedade, principalmente na promoção do uso racional de medicamentos, sendo o farmacêutico o profissional que tem como principal função a prevenção e promoção da saúde (Brasil, 2012), o objetivo deste trabalho foi analisar a responsabilidade social do farmacêutico a partir da experiência de pacientes atendidos em farmácia comunitária, identificando a percepção e as experiências destes pacientes com o atendimento farmacêutico ao longo de sua vida adulta e analisando tais percepções e experiências à luz de referenciais bioéticos que pautam a atuação socialmente responsável do farmacêutico enquanto profissional da saúde inserido no processo de cuidado.

A avaliação dos problemas relacionados a responsabilidade social do farmacêutico em farmácias comunitárias, foi realizada sob a ótica dos pacientes. A responsabilidade social' foi identificada neste trabalho de acordo com o princípio 14 da Declaração Universal sobre Bioética e Direitos Humanos, que preconiza a esse respeito, onde defende que a promoção da saúde e do desenvolvimento social em benefício dos respectivos povos é um objetivo fundamental dos governos que envolve todos os setores da sociedade (UNESCO, 2005).

\section{Método}

Trata-se de um estudo qualitativo, descritivo, exploratório, através de pesquisa de campo (Triviños, 1987), cuja função, longe de viabilizar qualquer pretensão de generalização ou inferência, o permitiu desvendar, analisar e discutir as implicações éticas relacionadas à responsabilidade social do farmacêutico a partir dos relatos de experiências de usuários de uma Farmácia Universitária do município de Curitiba. Para tanto, foram utilizados dois instrumentos. Primeiramente, fundamentado na classificação de Raynor3, foi elaborado um Protocolo de Registro de Atendimento Farmacêutico (Apêndice A) que categoriza em níveis o tipo de atendimento realizado e indica possíveis intervenções farmacêuticas. Há uma divisão de três níveis diferentes de 
orientação farmacêutica ao paciente, utilizando a comunicação verbal: nível 1: provisão de informação verbal breve centrada nas instruções posológicas, consistindo em reforçar informações sobre o nome e o propósito do medicamento, instruções sobre posologia e cuidados na administração; nível 2: explicação e conselhos sobre pontos principais da farmacoterapia do paciente, podendo requerer uma breve avaliação dos conhecimentos do paciente sobre a medicação; e nível 3: exploração completa envolvendo o uso de medicamentos pelo paciente. Nesta situação, paciente e farmacêutico analisam de modo completo aspectos do uso de medicamentos, incluindo atitudes, expectativas e experiências do paciente (Correr, Melchiors, Otuki, 2013).

A partir deste registro foram selecionados os atendimentos realizados nos níveis 1,2,3 a partir dos seguintes critérios de inclusão: pacientes maiores de 18 anos que se caracterizam no nível 1,2,3 da classificação do atendimento farmacêutico. Os pacientes foram convidados a conceder uma entrevista que teve por objetivo promover o compartilhamento das experiências dos mesmos nos processos de atuação farmacêutica durante sua vida adulta. A entrevista, realizada em uma sala reservada da Farmácia Universitária e registrada em áudio, seguiu um roteiro semiestruturado, apresentado na Figura 1, cuja delineação ocorreu a partir da problematização da responsabilidade social do farmacêutico enquanto profissional da saúde. No momento da entrevista também foram caracterizados dados sociodemográficos, gênero, idade, grau de escolaridade e bairro/cidade. A definição no número da amostra foi determinada qualitativamente por meio de técnica de saturação, ou seja, as entrevistas foram interrompidas após as respostas repetirem-se em seu núcleo de sentido.

As entrevistas tiveram duração de no máximo 10 minutos e foram realizadas entre o período de fevereiro a maio de 2016.Os dados foram analisados qualitativamente seguindo a abordagem da análise de conteúdo proposta por Bardin (Bardin, 2011), onde foram desdobradas a: pré-análise, descrição analítica e a interpretação inferencial. Na pré-análise, as entrevistas gravadas, foram transcritas, com a codificação dos pacientes (PCTE 1, PCTE 2...) para evitar a identificação dos mesmos, preservando assim o sigilo de suas identidades. Na segunda fase o material transcrito foi analisado classificando unidades temáticas em um processo denominado categorização, obtendo três categorias, organizadas de acordo com as experiências dos pacientes em relação a saúde, medicamentos/terapias e o profissional farmacêutico. O trabalho foi submetido ao Comitê de Ética da PUCPR, com o número CAAE 51242115.9.0000.0020, e todos os participantes registraram o consentimento após a 
explicação dos objetivos e implicações da pesquisa, os arquivos com as gravações das entrevistas ficarão arquivados em sigilo, sob guarda da pesquisadora responsável, e destruídos após cinco anos do término da pesquisa.

Figura 1. Roteiro Semi-estruturado

\section{Experiência com Saúde}

a. O que entende por "ter vida saudável"?

b. Possui alguma doença crônica diagnosticada?

\section{Experiência com Medicamentos/Terapias}

a. Utiliza algum medicamento de uso contínuo? Tais medicamentos foram por indicação médica?

b. Adere bem ao tratamento? Consegue lembrar de tomar todos, em todos os horários, todos os dias?

c. Sabe para que utiliza cada um deles? Quem explicou foi, o prescritor ou o farmacêutico ao dispensar?

d. Sabe qual o modo correto de utiliza-los?

e. Como se sente por ter que utilizar medicamentos?

f. Possui o hábito de se automedicar? Em que situações?

g. Considera benéfico terapias alternativas, como fitoterapia, acupuntura ou medidas não farmacológicas?

\section{Experiência com Farmacêutico}

a. Quando vai à farmácia, consegue identificar quem é o farmacêutico?

b. Tem conhecimento que é obrigatório a presença de um farmacêutico na farmácia em todo o período de funcionamento da mesma?

c. Quando vai à farmácia para comprar algum medicamento, seja com prescrição ou não, é sempre orientado quanto ao uso correto? Efeitos indesejáveis? Possíveis interações?

d. Se não orientado, parte de você solicitar essas informações?

e. Houve situações em que essas informações facilitaram a adesão ao tratamento? Ou dicas que auxiliaram a cumprir a terapia? 


\section{Resultados}

Como resultado das entrevistas, aplicadas por meio do roteiro semiestruturado, e segundo dados inseridos no protocolo de registro de atendimento farmacêutico, com os 15 pacientes participantes da pesquisa, obteve-se os seguintes dados sociodemográficos: em relação ao gênero, 9 se declaram feminino e 6 masculinos. Em relação à idade, 11 se apresentaram na faixa de 25-54 anos e 4 entre 55-64 anos. Quanto a escolaridade, 8 afirmaram ter apenas Ensino Médio completo e 7 Ensino Superior. Todos os participantes afirmaram morar em Curitiba, Paraná.

$\mathrm{Na}$ figura 2, apresentam-se dados obtidos segundo registros de atendimento farmacêutico, os quais indicam o motivo da procura pela Farmácia pelos entrevistados na época da pesquisa.

Figura 2. Tipo de Atendimento Farmacêutico procurado na Farmácia pelos entrevistados

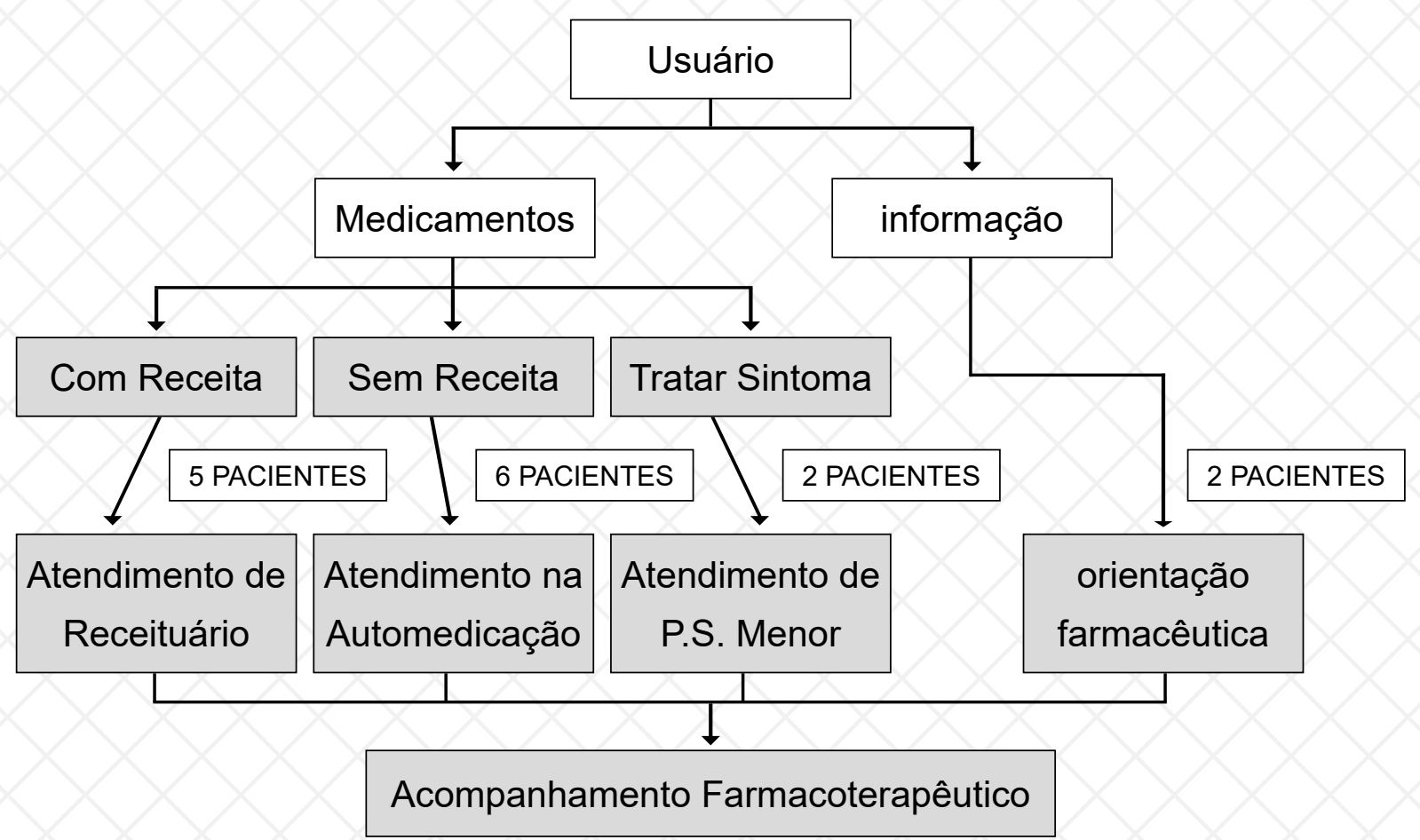

Em relação aos dados obtidos na entrevista semi-estruturada, na categoria "experiência com saúde", apresentados na Tabela 1, os entrevistados foram questionados a respeito do que entendiam sobre "vida saudável". Também foram indagados se possuíam alguma doença crônica e a maior parte dos pacientes relataram ter mais de uma doença diagnosticada, sendo a hipertensão a mais prevalente. 
Tabela 1. Categoria "experiência com saúde"

\section{EXPERIÊNCIA COM SAÚDE $\quad$ Relato dos pacientes}

\begin{tabular}{|c|c|}
\hline $\begin{array}{c}\text { O que entende por "ter vida } \\
\text { saudável"? }\end{array}$ & $\begin{array}{c}\text { "Uma vida saudável pra mim significa ter } \\
\text { uma alimentação saudável, atividade física e } \\
\text { cuidados com o corpo e mente." }\end{array}$ \\
\hline $\begin{array}{c}\text { Possui alguma doença crônica } \\
\text { diagnosticada? }\end{array}$ & "Sim, asma, hipertensão e problema na \\
tireoide."
\end{tabular}

A segunda categoria do roteiro semiestruturado agrupou questões relacionadas à experiência dos pacientes com medicamentos e terapias. Como a maior parte dos entrevistados apresenta alguma doença crônica, foi natural encontrar que a maioria faz uso medicamentos de uso contínuo. As únicas exceções foram no tratamento da doença Psoríase, onde o paciente declara utilizar apenas em forma tópica em momentos de crise, e da doença Artrose, onde o paciente alega usar medicamento apenas quando apresenta dor intensa. Os pacientes também foram questionados quanto à adesão ao tratamento, orientações a respeito da terapia medicamentosa e automedicação. Outra experiência relacionada a tratamento identificada no instrumento foi o uso de terapias alternativas, como fitoterapia, acupuntura ou medidas não farmacológicas. As três práticas complementares mais citadas foram acupuntura, fitoterapia e homeopatia, as quais são áreas de atuação do farmacêutico, asseguradas por resoluções do Conselho Federal de Farmácia. A síntese dos resultados desta categoria encontra-se na Tabela 2.

Tabela 2. Categoria "experiência com medicamentos e terapias"

\begin{tabular}{|c|c|}
\hline EXPERIÊNCIA COM & Relato dos pacientes \\
MEDICAMENTOS/TERAPIAS & \\
\hline $\begin{array}{c}\text { Utiliza algum medicamento } \\
\text { de uso contínuo? Tais } \\
\text { medicamentos foram por } \\
\text { indicação médica? }\end{array}$ & "Uso sim. Sim, todos por indicação médica." \\
\hline $\begin{array}{c}\text { Adere bem ao tratamento? } \\
\text { Consegue lembrar de tomar } \\
\text { todos, em todos os horários, } \\
\text { todos os dias? }\end{array}$ & $\begin{array}{c}\text { "Isso eu consigo, porque é de manhã antes do } \\
\text { cafe então quando eu chego em casa } \\
\text { eu tomo o medicamento." }\end{array}$ \\
\hline
\end{tabular}



deles? Quem explicou foi, o prescritor ou o farmacêutico ao dispensar? Sabe qual o modo correto de utiliza-los?
Sabe para que utiliza cada um

"Sei sim pra que serve. Foi o farmacêutico. Ele que me deu todas as explicações. O médico corre muito então não tem tempo de ficar te explicando muito, então quando você vai comprar o remédio o farmacêutico explica tudo certinho. Já tentei até fazer manipulado com a orientação do farmacêutico." "Sim o médico me explicou. Todos eles eu tomo pela manhã, de preferência em jejum com água."

Como se sente por ter que utilizar medicamentos?

"Eu não me sinto confortável, mas não tenho escolha. Eu tenho que usar, mas preferiria não ter que usa-los."

"Acho que todo mundo tem né, principalmente com dor. Mas medicamento que uso é tipo "diclofenaco", um "buscopan" para dor de

Possui o hábito de se automedicar? Em que situações?

no rim, se automedicar nessa proporção não. Mas

o básico. Mas minha esposa as vezes passa com dor mas não toma, sem passar pelo médico."

"Sim, faço uso de todos. Tomo florais, tomei por muito tempo homeopáticos e faço acupuntura, eu faço e vejo muito resultado. Para minha enxaqueca inclusive foi a acupuntura que

Considera benéfico terapias alternativas, como fitoterapia, acupuntura ou medidas não farmacológicas? resolveu. Na primeira sessão, já fazia 20 dias que estava com enxaqueca diária, já diminuiu. Eu sentia as agulhas pulsando na minha testa e quando ele tirou parece que tirou com a mão a dor. E quanto a fitoterapia eu tomo muito chá pra tudo, para cansaço, stress, para dormir, para cólica. Tomamos muito."

$\mathrm{Na}$ categoria seguinte, foram selecionadas perguntas que revelassem experiências com o profissional farmacêutico. As perguntas tinham o objetivo de descobrir qual o conhecimento dos pacientes em relação a identificação do farmacêutico na farmácia comunitária e a obrigatoriedade da presença do mesmo em todo período de funcionamento. 
Além da identificação e da presença do profissional, os pacientes também foram questionados quanto a orientação e indicação farmacêutica. Os entrevistados mencionaram que dificilmente recebem orientação na farmácia quando adquirem medicamento, seja com ou sem prescrição, ou recebem apenas se os mesmos solicitarem. Ao mesmo tempo que os entrevistados relataram ter que solicitar orientações quanto ao uso correto, possíveis interações e efeitos indesejáveis, declararam também que as orientações já recebidas alguma vez facilitaram a adesão ao tratamento. Além de orientações, os pacientes declararam que já buscaram na farmácia a indicação de algum tratamento para sintomas mais simples. Em relação a outros serviços farmacêuticos como verificação de pressão arterial e glicemia capilar, os mesmos não são utilizados pelos pacientes entrevistados em farmácias. Assim como o serviço de Atenção Farmacêutica, onde a maioria dos entrevistados não conhecem ou ouviram falar do serviço. O apanhado geral da categoria está apresentado na Tabela 3.

Tabela 3. Categoria "experiência com farmacêutico"

\begin{tabular}{|c|c|}
\hline $\begin{array}{c}\text { EXPERIÊNCIA COM } \\
\text { FARMACÊUTICO }\end{array}$ & Relato dos pacientes \\
\hline $\begin{array}{l}\text { Quando vai à farmácia, } \\
\text { consegue identificar quem é o } \\
\text { farmacêutico? }\end{array}$ & $\begin{array}{c}\text { "Depende da farmácia, porque algumas têm } \\
\text { muitos atendentes e só dá para saber se você } \\
\text { perguntar." }\end{array}$ \\
\hline $\begin{array}{l}\text { Tem conhecimento que é } \\
\text { obrigatório a presença de um } \\
\text { farmacêutico na farmácia em } \\
\text { todo o período de funcionamento } \\
\text { da mesma? }\end{array}$ & $\begin{array}{c}\text { "Sim. Inclusive já fui embora de uma farmácia } \\
\text { porque não tinha a presença do farmacêutico e } \\
\text { eu sei que é obrigatório." }\end{array}$ \\
\hline $\begin{array}{l}\text { Quando vai à farmácia para } \\
\text { comprar algum medicamento, } \\
\text { seja com prescrição ou não, é } \\
\text { sempre orientado quanto ao uso } \\
\text { correto? Efeitos indesejáveis? } \\
\text { Possíveis interações? Se não } \\
\text { orientado, parte de você solicitar } \\
\text { essas informações? }\end{array}$ & $\begin{array}{l}\text { "Nunca. Se comprei } 100 \text { remédios, em } 100 \text { não } \\
\text { recebi orientação. Só se eu pedir." " Somente } \\
\text { em casos de dúvida. Se me restou alguma } \\
\text { dúvida sobre armazenamento ou modo de uso ai } \\
\text { eu pergunto.” }\end{array}$ \\
\hline
\end{tabular}




\begin{tabular}{|c|c|}
\hline $\begin{array}{c}\text { Houve situações em que essas } \\
\text { informações facilitaram a adesão } \\
\text { ao tratamento? Ou dicas que } \\
\text { auxiliaram a cumprir a terapia? }\end{array}$ & $\begin{array}{c}\text { "Sim. Quando eu operei, fiz minha bariátrica, } \\
\text { todas as orientações que eu me informei com o } \\
\text { farmacêutica, tudo o que ela passou para mim } \\
\text { me ajudou muito na minha melhora e no meu } \\
\text { desenvolvimento, no decorrer do tratamento. Fui } \\
\text { muito bem orientada por ela." }\end{array}$ \\
\hline $\begin{array}{c}\text { Houve situações em que foi até } \\
\text { a farmácia solicitando indicação } \\
\text { de algum tratamento para tratar } \\
\text { sintomas mais simples? }\end{array}$ & $\begin{array}{r}\text { "Sim, e foi aqui na farmácia também. A indicação } \\
\text { foi, por exemplo, para torcicolo, colocar o } \\
\text { "Salonpas", fazer uma compressa" }\end{array}$ \\
\hline $\begin{array}{c}\text { Possui o hábito de verificar } \\
\text { pressão arterial e /ou glicemia } \\
\text { capilar na farmácia? }\end{array}$ & $\begin{array}{r}\text { "Eu sempre acompanhei pressão. Eu tenho em casa mas vou verificar em farmácia } \\
\text { quando sinto que estou meio diferente." }\end{array}$ \\
\hline $\begin{array}{c}\text { Conhece ou já ouviu falar } \\
\text { do Serviço de Atenção } \\
\text { Farmacêutica? }\end{array}$ & \\
\hline
\end{tabular}

Comparando os dois instrumentos utilizados no estudo percebemos que, os pacientes que buscaram a farmácia solicitando a indicação de algum medicamento para tratar algum sintoma menor de saúde, corresponde com as respostas obtidas no roteiro semiestruturado. Os pacientes relataram que:

Sim, teve uma vez que o meu filho teve uma dor na barriga muito forte, que a gente não sabia o que fazer, e foi a indicação de um remedinho super bom que eu tenho até guardado o nome de tão boa que foi a indicação.

Sim, teve uma vez que estava com o peito carregado, e foi me indicado fazer nebulização com soro fisiológico.

Os pacientes que foram até a farmácia em busca apenas de informação, relataram:

Dificilmente, essas dúvidas normalmente eu tiro com o médico. Aqui na Farmácia PUC, como eu sou mais intima de vocês, eu pergunto antes de fazer alguma mudança, mas em outras farmácias não. Você acaba não confiando no profissional por não conhecer. 
Ao serem questionados sobre automedicação, o grupo de pacientes que solicitou 0 atendimento com prescrição médica se mostraram resistentes ao ato.

Eventualmente se tiver um mal estar ou uma coisa pequena, sim.

Não. Mesmo assim eu não uso. Prefiro não usar. Às vezes eu tomo um chá natural que ajuda aliviar.

Por fim, o atendimento dos pacientes que solicitaram apenas o nome do medicamento, sem apresentar prescrição, quando questionados se tinham conhecimento para que usavam cada medicamento, todos afirmaram que sim. Ao perguntar quem forneceu a explicação, as respostas se dividiram entre: o médico, médico e farmacêutico, apenas o farmacêutico e equipe multidisciplinar.

\section{Discussão}

A maior parte dos participantes desta pesquisa foram mulheres, o que corresponde ao achado de Tavares et al. (Tavares et al., 2015) em estudo que mostra prevalência de uso de medicamentos para hipertensão arterial entre mulheres, na comparação com homens, atribuindo ao fato de mulheres utilizarem mais serviços e terem maiores cuidados com a saúde (Tavares et al., 2015). Esses resultados corroboram as preocupações com o autocuidado do homem em nossa sociedade. Braz (2005), analisou, a partir da bioética, a construção da subjetividade masculina e seu impacto sobre a saúde do homem, e, considerando o princípio de justiça distributiva, constatou-se que a saúde do homem tem sido pouco considerada pelas políticas públicas de saúde. Frente aos nossos resultados, deve ficar registrada, portanto, a reponsabilidade social do farmacêutico na redução de tais injustiça, dedicando uma maior atenção no atendimento da população masculina, que deve ser constantemente informada sobre a importância do autocuidado em saúde.

Quanto ao uso de medicamentos, o expressivo relato de utilização de medicamentos de uso contínuo corrobora os resultados da Pesquisa Nacional de Saúde de 2013, no Brasil, a prevalência do uso de medicamentos para Doenças Crônicas Não Transmissíveis (DCNT) como hipertensão arterial, diabetes e asma. Importa destacar que embora essas DCNTs sejam mais comuns entre os idosos, a grande maioria dos pacientes participantes da pesquisa não se enquadram na categoria de idade considerados "idosos". A doença crônica mais prevalente nesta pesquisa foi hipertensão, embora o objetivo não fosse avaliar a efetividade dos tratamentos e/ou controle, nenhum paciente relatou problemas atuais em relação a doença (Menguel, 2015). 
De acordo com a OMS, "saúde é o estado de completo bem-estar físico, mental e social e não apenas a ausência de doença" (WHO, 1948). No presente estudo, foi observado que os entrevistados também adotam essa ideia, uma vez que associam "vida saudável" à alimentação e exercícios físicos, mesmo a grande maioria apresentando doença crônica diagnosticada e fazendo uso contínuo de medicamentos. Segundo Lafévre (Leite, Veber, 2004), existe também outra linha de pensamento sobre ser saudável, relacionando a ideia de saúde ao consumo de medicamentos, tornando-os símbolos de saúde. Neste sentido, pesquisa realizada por Fleith et al. (Fleith et al., 2008) mostrou que a maioria dos participantes fazia uso de medicamentos, mas mesmo assim relatavam estar com saúde, demonstrando assim a simbologia do medicamento em relação a saúde (WHO, 1948). Segundo outro estudo, cerca de $40 \%$ dos pacientes atendidos nos serviços de saúde básica não precisam de medicamentos, por não manifestar nenhum indicativo de doença orgânica ou psíquica, ainda assim, saem das consultas com prescrição de medicamentos (Santi, 2016). Em razão disso, a necessidade de quebra de paradigma, considerando qualidade de vida também relações pessoais e sociais, contribuindo para o equilíbrio dos níveis de saúde, tal como os recursos terapêuticos (Semplici, 2010).

Potter (Potter, 1971) defende que o manejo da vida através substâncias químicas apresenta riscos. Ao mesmo tempo em que a ciência avança na cura dos males do mundo, o mesmo avanço pode trazer consequências indesejáveis e incalculáveis. O progresso do conhecimento não se limita a destruição de pragas, vírus ou células cancerosas, mas influencia também na elaboração de substâncias químicas que influenciam comportamentos psicológicos e emocionais dos seres humanos (Potter, 1971). Com isso, nos deparamos ao que é chamado de "medicalização da vida", com a medicina presente em todas as etapas da vida humana, com o adverso da comercialização, transformando tudo que é relativo a vida ou saúde, em mercadoria e dinheiro (Drummond, 2016).

Uma das maneiras de reduzir problemas relacionados a medicamentos é por meio da melhoria da qualidade tanto da prescrição quanto da dispensação de medicamentos. Essa qualidade pode ser alcançada com a ação conjunta entre prescritores e farmacêuticos em prol do paciente. Deve ser identificado dificuldades que possam levar pacientes a não cumprirem o tratamento e deve-se oferecer todas as informações necessárias para que o uso racional ocorra e para que os pacientes compreendam a forma mais apropriada do uso dos medicamentos prescritos (Fleith et al., 2008). 
Segundo a OMS, adesão ao tratamento "é a medida com que o comportamento de uma pessoa - tomar a sua medicação, seguir a dieta e/ ou mudar seu estilo de vida - corresponde às recomendações de um profissional de saúde" (WHO, 2003). A adesão acontece a partir do momento em que há uma administração segura do medicamento. O paciente deve participar deste processo empoderando-se de sua terapia, tendo maior controle sobre decisões e ações que afetam sua saúde. Uma vez que o paciente compreende seu papel no cuidado de sua saúde, problemas relacionados a medicamentos podem ser evitados (Gimenes, 2016).

No Brasil, o uso incorreto de medicamentos deve-se comumente a: polifarmácia, uso indiscriminado de antibióticos, prescrição não orientada por diretrizes, automedicação inapropriada entre outros (Brasil, 2012). No tocante ao uso racional de antimicrobianos, embora existam legislações que regulamentam o uso correto e a prescrição dos mesmos, o controle obtido ainda tem seu êxito parcial. E mais uma vez, temos o profissional farmacêutico podendo contribuir para tal ação, conscientizando cada vez mais a população do uso adequado desta classe de medicamentos (Sampaio, Sancho e Lago,2018). Analisando a representação da medicamentação na sociedade brasileira sob a perspectiva da Bioética, Palodeto e Fischer (2018) concluíram que, embora a maioria dos participantes tenha afirmado se considerar saudável, mais da metade informou consumir medicamento de uso contínuo, o que leva à expectativa de que o uso de medicamentos está naturalizado e incorporado a uma compreensão de vida saudável.

Segundo Aquino, Barros e Silva (Aquino, Barros, Silva, 2010), a prática da automedicação no Brasil é proporcional a $35 \%$ do consumo dos medicamentos, independente de classe social. Ela é justificada pelo fácil acesso a medicamentos obtidos em drogarias sem prescrição médica e pela dificuldade de ter atendimento médico no Sistema Único de Saúde (Santos, Freitas, Eduardo, 2015). Os medicamentos mais usados por automedicação são os analgésicos e anti-inflamatório (Arrais et al., 1997) (Bertoldi et al., 2004). Assim como Arrais et al. (Arrais et al., 1997) e Bertoldi et al. (Bertoldi et al., 2004), também apontaram os analgésicos como os medicamentos mais utilizados em seus estudos com $21,6 \%$ e $26,6 \%$, respectivamente $15,17,18$. Mesmo sendo considerada uma prática de autocuidado, a automedicação pode ter um grande potencial danoso à saúde, uma vez que nenhum medicamento é inofensivo ao organismo (Schuelter-Trevisol et al., 2011). O uso incorreto de substâncias consideradas "inócuas" pela população pode ocasionar diversas consequências, tais como hipersensibilidade, sangramento do trato digestivo, dependência e até masca- 
rar um transtorno de saúde maior, com o alívio momentâneo dos sintomas podendo agravar o quadro clínico de alguma doença (Forner, Silva, Brzozoeski, 2008).

O homem também sempre buscou na natureza meios para aumentar as chances de sobrevivência e melhoria da qualidade de vida. A realidade sociocultural de cada região é uma grande influência nas práticas de cuidado à saúde, inclusive, o Brasil resgata antigos costumes da medicina tradicional chinesa além de práticas populares do país (Matsuchita, Matsuchita, 2015). Segundo dados da OMS, em países em desenvolvimento, em torno de $80 \%$ da população utilizam mais práticas tradicionais nos cuidados de saúde do que as convencionais. Ao final da década de 1970, a OMS criou o Programa de Medicina Tradicional com objetivo de estimular a promoção de tais terapias e inseri-las no sistema de saúde. No Brasil, o Ministério da Saúde aprovou em 2006 a Política Nacional de Práticas Integrativas e Complementares, que valoriza ao mesmo tempo a humanização do cuidado e respeito ao pluralismo cultural (Brasil, 2015). A esse respeito, no presente estudo, pacientes responderam se "consideram benéfico terapias alternativas, como fitoterapia, acupuntura ou medidas não farmacológicas", sendo favoráveis ao uso.

De acordo com a Resolução $n^{\circ} 357$ de 2001 do CFF, que dispõe sobre as Boas Práticas de Farmácia, estabelece que "a presença e atuação do farmacêutico é requisito essencial para a dispensação de medicamentos aos pacientes, cuja atribuição é indelegável, não podendo ser exercida por mandato nem representação". Os dados obtidos na presente pesquisa constataram uma possível deficiência na dispensação de medicamentos, mas especificamente na orientação sobre o uso correto. Além de orientações, o profissional farmacêutico também está apto a atuar na indicação de tratamento para transtornos menores de saúde, dentro do arsenal terapêutico de medicamentos isentos de prescrição. Os entrevistados mencionaram que dificilmente recebem orientação na farmácia quando adquirem medicamento, seja com prescrição ou não, o que mostra a exposição em que os pacientes se encontram ao uso incorreto de medicamentos.

Oliveira (2015), tecendo considerações acerca da atenção farmacêutica, defende que cada vez mais a atividade nessa prática perpassará pela necessidade de rever os conceitos pessoais e profissionais do Farmacêutico com um balizamento da Ética. Desde um aspecto normativo, existem diversos documentos e resoluções dos órgãos competentes que regulamentam a prestação de serviços farmacêuticos em farmácia e a exigência da prática de atenção farmacêutica como parte do manual de Boas 
Práticas de Farmácia. Em 11 de agosto de 2014, foi sancionada pela Presidência da República e publicada a Lei no 13.021/14 que muda o conceito e classificação de farmácias no Brasil reconhecendo-as como Estabelecimento de Saúde (Brasil, 2001). A Lei n $n^{\circ} 13.021$ regulamenta a Assistência Farmacêutica, Assistência à Saúde e Orientação Sanitária individual e coletiva. Ainda assim, os dados obtidos nas entrevistas levantam a suspeita de que estes serviços farmacêuticos não são utilizados pela população, seja por falta de conhecimento ou por conveniência.

Os resultados obtidos na atual pesquisa permitiram analisar questões relacionadas aos princípios que compõe a Declaração Universal sobre Bioética e Direitos Humanos (DUBDH). Este documento foi adotado durante a $33^{\mathrm{a}}$ Conferência Geral das Nações Unidas para Educação, a Ciência e a Cultura (UNESCO), em outubro de 2005, revelou um olhar bioético voltado também para questões sociais, sanitárias e ambientais.

Ao abordar tais questões éticas, a Declaração conduz ao respeito pela dignidade humana, pelos direitos humanos; ao combate à pobreza, violência; ao acesso universal a saúde, equidade; a qualidade de vida, da água e da biodiversidade (UNESCO, 2005). Quando mencionado o princípio de Dignidade Humana e Direitos Humanos, os interesses e o bem-estar do paciente devem prevalecer sobre o interesse exclusivo da ciência ou da sociedade. Bem-estar está associado a vida saudável, embora os participantes não tenham definido de tal maneira. Boa alimentação e exercícios físicos de qualidade, tendem a melhorar o estado de saúde do indivíduo, constituindo um dos direitos fundamentais do ser humano (UNESCO, 2005).

No que diz respeito ao princípio Responsabilidade Social e Saúde, destaca-se o acesso a cuidados de saúde de qualidade e aos medicamentos essenciais, uma vez que a saúde é essencial à própria vida e deve ser considerada um bem social e humano (UNESCO, 2005), visto que a garantia do acesso aos medicamentos essenciais é considerada uma obrigação indelegável do Estado, sendo pauta permanente na agenda de diversos países e organizações internacionais como Organização das Nações Unidas, que apresenta como meta especificamente a necessidade de "promover o acesso a medicamentos essenciais nos países em desenvolvimento" (Bergel, 2007). A grande parte dos entrevistados utiliza medicamentos de uso contínuo, seja por aquisição pela rede pública ou privada, o que mais uma vez corrobora com a realidade de países em desenvolvimento, que embora disponham de lista de medicamentos essenciais, nem sempre há disponibilidade dos mesmos em unidade de saúde pública, forçando os pacientes a comprar nas redes privadas (UNESCO, 2010). 
Diante desta situação, a presença de um profissional capacitado dispensando o medicamento é primordial para a garantia da obtenção de melhores resultados clínicos. A orientação quanto ao uso correto de medicamentos, efeitos indesejáveis ou possíveis interações, fornece ao paciente informações que otimizarão a terapia, auxiliando principalmente na adesão ao tratamento. Além disso, com o farmacêutico exercendo serviços clínicos inerentes da profissão, como a prescrição farmacêutica de algum medicamento isento de prescrição para tratar algum sintoma de transtorno de saúde menor, ou verificação de parâmetros fisiológicos como pressão arterial e glicemia capilar, garantem ao paciente o acesso a cuidados de saúde, assegurando a promoção, proteção e recuperação da saúde além do uso racional de medicamentos.

A autonomia das pessoas no que respeita à tomada de decisões, desde que assumam a respectiva responsabilidade e respeitem a autonomia dos outros, deve ser respeitada. No caso das pessoas incapazes de exercer a sua autonomia, devem ser tomadas medidas especiais para proteger os seus direitos e interesses (UNESCO, 2005). Vários fatores influenciam o cumprimento e resultados obtidos de algum tratamento, porém, o conhecimento, por parte do paciente, do medicamento ou terapia utilizada é essencial para que ele entenda importância do uso, conheça os riscos e compreenda a razão pelo qual está tendo que usar o medicamento.

Na pesquisa presente, foi observado esse interesse pois, além de todos os participantes alegarem saber para que utiliza cada medicamento, seja por orientação do médico ou farmacêutico, eles também alegaram que quando não orientados quanto ao uso correto, solicitam ou vão em busca dessas informações. A automedicação também é uma prática onde deve ser respeitada a autonomia do paciente.

De acordo com OMS, automedicação é "a seleção e o uso de medicamentos por pessoas para tratar doenças auto- diagnosticadas ou sintomas e deve ser entendida como um dos elementos do auto-cuidado". Ainda, segundo a OMS "o auto-cuidado é o que as pessoas fazem por si mesmas para estabelecer e manter a saúde, prevenir e lidar com a doença. É um conceito amplo, que abrange: higiene (geral e pessoal), nutrição (tipo e qualidade do alimento ingerido), estilo de vida (atividade física, lazer, etc.); fatores ambientais (condições de vida, hábitos sociais, etc.); fatores sócio-econômicos (nível de renda, crenças culturais, etc.); automedicação" (WHO, 2001).

Porém, mesmo sendo considerada uma prática de autocuidado, a automedicação pode ter um grande potencial danoso à saúde, uma vez que nenhum medicamento é inofensivo ao organismo (Forner, Silva, Brzozoeski, 2008). Neste caso, o respeito 
pela tomada de decisão do paciente acontece desde que o mesmo assuma a responsabilidade pelos seus atos. No entanto, cabe ao farmacêutico orientar os possíveis riscos/ benefícios, assim o paciente terá informações suficientes para decidir.

Por fim, a população está exposta a uma sociedade de mercado onde algumas doenças são desenvolvidas a partir de uma rotina opressora, tornando o ser humano em mais uma forma de atingir lucros. Serviços de saúde que garantem qualidade de vida, quando mercantilizados, ferem o princípio fundamental dos direitos humanos que é o direito a saúde. Reconhecer saúde como qualidade de vida, tal como defende a DUBDH, nos faz refletir sobre as desigualdades no acesso aos bens e serviços e como a atuação farmacêutica pode contribuir. O resgate do cuidado, neste caso o cuidado farmacêutico, tem a finalidade de avaliar o uso de novas tecnologias, adotando uso racional das mesmas, recuperando e promovendo saúde, prevenindo doenças que podem ser evitadas, auxiliando no desenvolvimento de condições de vida mais saudáveis.

\section{Considerações Finais}

Embora os relatos dos pacientes tenham revelado um farmacêutico comprometido com suas funções, algumas questões encontradas a partir da percepção e experiências com atendimento farmacêutico indicam a necessidade de maior atenção ao princípio da responsabilidade social por parte do profissional. Os relatos, ainda que pontuais, indicam experiências farmacêuticas onde houve pouca informação, orientação e monitoramento que podem prejudicar de modo geral a saúde do paciente, e de modo específico, a farmacoterapia.

No Brasil, o principal campo de trabalho dos farmacêuticos é em farmácias comunitárias. Essa realidade de atuação convive com várias questões éticas, proveniente da dificuldade de realizar a prática farmacêutica em um local comercial. No entanto, a comunidade tem necessidades em relação ao uso de medicamentos, e devem ser atendidas da melhor forma a fim de utiliza-los como recurso terapêutico de modo correto e racional.

Ainda que as experiências relatadas nesta pesquisa apresentem um farmacêutico presente e ativo na sociedade, demonstrando o avanço obtido nos últimos anos na qualidade de serviços farmacêuticos, ainda há aspectos importantes a serem considerados na atuação socialmente responsável destes profissionais, especificamente no que se refere aos serviços clínicos, como orientação e acompanhamento farma- 
coterapêutico, que busca otimização dos resultados terapêuticos, desenvolvendo hábitos de saúde, aumentando a qualidade de vida da população.

Uma maior aproximação entre a formação e prática farmacêutica com a bioética pode auxiliar a preencher lacunas, buscando pautar questões que ocorrem na realidade das farmácias comunitárias e que podem repercutir positivamente tanto na qualidade de vida dos pacientes quanto na segurança da atuação profissional.

\section{Referências}

1. Aquino, DS, Barros, JAC, Silva, MDP. A automedicação e os acadêmicos da área de saúde. Ciênc. saúde coletiva. 2010; 15(5):2533-2538.

2. Arrais, PSD, Coelho, HLL, Batista, MCDS, Carvalho, ML, Righi, RE, Arnau, JM. Perfil da Automedicação no Brasil. Rev Saúde Pública 1997; 31(1):71-77.

3. Bardin, L. Análise de conteúdo. Tradução Luís Antero Reto e Augusto Pinheiro. São Paulo: Edições 70, 2011.

4. Bergel, SD. Responsabilidad social y Salud. Revista Latinoamericana de Bioética 2007; 7(12):10-27.

5. Bertoldi, AD, Barros, AJD, Hallal, PC, Lima, RC. Utilização de medicamentos em adultos: prevalência e determinantes individuais. Rev Saúde Pública 2004; 38:228-238.

6. Brasil. Conselho Federal de Farmácia. Resolução 357 de 20 de abril de 2001 . Diário Oficial da União. Brasília: 2001.

7. Brasil. Ministério da Saúde. Secretaria de Atenção à Saúde. Departamento de Atenção Básica. Política nacional de práticas integrativas e complementares no SUS: atitude de ampliação de acesso - Brasília: Ministério da Saúde, 2015.

8. Brasil. Ministério da Saúde. Secretaria de Ciência, Tecnologia e Insumos Estratégicos. Uso racional de medicamentos: temas selecionados - Brasília: Ministério da Saúde, 2012.

9. Brasil. Ministério da Saúde. Secretaria de Ciência, Tecnologia e Insumos Estratégicos. Uso racional de medicamentos: temas selecionados - Brasília: Ministério da Saúde, 2012.

10. Braz, M. A construção da subjetividade masculina e seu impacto sobre a saúde do homem: reflexão bioética sobre justiça distributiva. Ciência \& Saúde Coletiva, 10(1):97-104, 2005

11. Cipolle, RJ, Strand, LM, Morley, PC. O exercício do cuidado farmacêutico. Revisão técnica: Conselho Federal de Farmácia, 2006.

12. Correr, CJ, Melchiors, AC, Otuki, MF. A Prática Farmacêutica na Farmácia Comunitária. Porto Alegre: [s.n.], 2013. 
13. Drummond, JP. Bioética, dor e sofrimento. Cienc. Cult. [serial on the Internet]. 2011 [cited 2016 Nov 15]; 63(2): 32-37. Available from: http://cienciaecultura.bvs. br/scielo.php?script=sci_arttext\&pid=S0009-67252011000200011\&lng=en.

14. Fleith, VD, Figueiredo, MA, Figueiredo, KFLRO, Moura, EC. Perfil de utilização de medicamentos em usuários da rede básica de saúde de Lorena, SP. Ciênc. saúde coletiva. 2008; 13:755-762.

15. Forner, S, Silva, MS, Brzozowski, FS. Propaganda de medicamentos, automedicação e a ética farmacêutica: uma tríade farmacêutica. Artigo de revisão. Instituto Sallus, 2008.

16. Gimenes, FRE. Administração: não basta usar, é preciso conhecer a maneira correta. Uso Racional de Medicamentos: fundamentação em condutas terapêuticas e nos macroprocessos da Assistência Farmacêutica 2016; 1(18).

17. Leite, SN, Vieira, M, Veber, AP. Estudos de utilização de medicamentos: uma síntese de artigos publicados no Brasil e América Latina. Ciênc. saúde coletiva. 2004; 13:793-802.

18. Matsuchita, HLP, Matsuchita, ASPA.Contextualização da Fitoterapia na Saúde Pública. Uniciências 2015; 19(1): 86-92.

19. Menguel, SS, Tavares, NUL, Costa, KS, Malta, DC, Júnior, JBS. Fontes de obtenção de medicamentos para tratamento de hipertensão arterial no Brasil: análise da Pesquisa Nacional de Saúde, 2013. Rev. bras. epidemiol. 2015; 18 (2): 192203.

20. Molina, LR, Finkler, M. Ética e prática profissional farmacêutica: um panorama das discussões em pauta. Bioética e Saúde Pública 2016; 1:145-163.

21. Oliveira, NA. Atenção Farmacêutica: considerações éticas na relação do profissional de saúde com o usuário de medicamento. Tempus, actas de saúde colet, Brasília, 9(2), 29-40, jun, 2015

22. Palodeto, MFT; Fischer, ML. A representação da medicamentação sob a perspectiva da Bioética. Saúde Soc. São Paulo, v.27, n.1, p.252-267, 2018.

23. Potter, V. R. Bioethics: bridge to the future. Prentice Hall, Englewood Cliffs (NJ), 1971.

24. Sampaio, PS, Sancho, LG, Lago, RF. Implementação da nova regulamentação para prescrição e dispensação de antimicrobianos: possibilidades e desafios. Cad. Saúde Colet. 2018, 26 (1): 15-22.

25. Santi, LQ. Prescrição: o que levar em conta? Uso Racional de Medicamentos: fundamentação em condutas terapêuticas e nos macroprocessos da Assistência Farmacêutica 2016; 1(14).

26. Santos, PNM, Freitas, RF, Eduardo, AMLN. Automedicação infantil: conhecimento e motivação dos pais. Revista Multitexto 2015; 3(1).

27. Schuelter-Trevisol, F, Trevisol, DJ,Ung, GS, Jacobowski, B. Automedicação em 
universitários. Rev Bras Clin Med 2011; 9(6):414-7.

28. Semplici,S. El "más alto nivel posible". Un derecho assimétrico. Acta Bioethica 2010; 16 (2): 133-141.

29. Tavares, NUL, Costa, KS, Vieira, MLFP, Malta, DC, Júnior, JBS. Uso de medicamentos para tratamento de doenças crônicas não transmissíveis no Brasil: resultados da Pesquisa Nacional de Saúde, 2013. Epidemiol. Serv. Saúde 2015; 24(2): 315-323.

30. Triviños, ANS. Introdução à pesquisa em ciências sociais: a pesquisa qualitativa em educação: o positivismo, a fenomenologia, o marxismo. Atlas 1987, 175p.

31. Unesco. Declaração Universal sobre Bioética e Direitos Humanos. 2005. Acessível em https://unesdoc.unesco.org/ark:/48223/pf0000146180_por

32. Unesco. Report of the international bioethics committee of Unesco on social responsibility and health. 2010. Acessível em http://www.atds.org.tn/Rapport_ComitelntBioethique.pdf

33. Vianal, KP, Brito, AS, Rodrigues, CS, Luiz, RR. Acesso a medicamentos de uso contínuo entre idosos, Brasil. Rev Saúde Pública 2015; 49:14.

34. World Health Organization (WHO). Adherence to long-term therapies: evidence for action. Geneva: World Health Organization; 2003.

35. World Health Organization (WHO). Official Records of the World Health Organization, ${ }^{\circ}{ }^{\circ}$ 2, p. 100 . United Nations, World Health Organization. Geneve, Interim Comission; 1948.

36. World Health Organization. The Rational use of drugs: report of the conference of experts. Nairóbi, 25-29 november 1985. Geneva: WHO; 1987.

37. World Health Organization (WHO). The role of the pharmacist in self-medication and self-care. Genebra: WHO; 1998. (WHO/DAP/09.13).

Recebido: 29/04/2019. Aprovado: 05/08/2019.

\section{Apêndice A - Protocolo de Registro de Atendimento Farmacêutico}

Etapas do processo de Dispensação

1. Paciente chegou a farmácia procurando por medicamento ou informação?

( ) sim ( ) não Observações:

2. Se a procura foi por INFORMAÇÃO, qual a dúvida do paciente?

( ) sim ( ) não Observações:

a. Foi possível orientar imediatamente? Se sim, qual a orientação dada?

( ) $\operatorname{sim}$ ( ) não Observações:

b. Se não, foi tomada outras providências? Se sim, quais?

( ) sim ( ) não Observações: 
3. Se a procura foi por MEDICAMENTO, havia prescrição?

( ) sim ( ) não Observações:

a. Se sim, houve análise dos dados?

( ) $\operatorname{sim}($ ) não Observações:

b. Medicamento prescrito está adequado de acordo com as características do paciente?

( ) sim ( ) não Observações:

c. Se inadequado, foi recomendado entrar em contato com o prescritor?

( ) sim ( ) não Observações:

d. É a primeira vez que o paciente vai utilizar o medicamento?

( ) $\operatorname{sim}$ ( ) não Observações:

e. Se sim, o medicamento foi dispensado orientando o uso?

( ) $\operatorname{sim}$ ( ) não Observações:

f. Efeito esperado?

( ) sim ( ) não Observações:

g. Duração do tratamento?

( ) $\operatorname{sim}$ ( ) não Observações:

h. Interações?

( ) sim ( ) não Observações:

i. Reações Adversas?

( ) sim ( ) não Observações:

j. Foi dispensado o medicamento que estava prescrito ou o genérico ou similar?

( ) $\operatorname{sim}$ ( ) não Observações:

k. O paciente solicitou alguma orientação/dúvida para além as acimas informações? Se sim, quais?

( ) $\operatorname{sim}$ ( ) não Observações:

I. Se não for a primeira vez do uso, foi avaliado a efetividade e segurança do medicamento?

( ) sim ( ) não Observações:

m. O uso de tal medicamento está sendo efetivo e seguro?

( ) $\operatorname{sim}$ ( ) não Observações:

n. Se foi detectado que o uso está sendo inefetivo e/ou inseguro, foi possível uma orientação imediata? Se sim, quais foram as orientações dadas?

( ) $\operatorname{sim}($ ) não Observações:

o. Se não, foi tomada outras providências para a resolução do problema?

( ) $\operatorname{sim}$ ( ) não Observações:

p. Foram repassadas informações complementares como medidas não farmacológicas?

( ) sim ( ) não Observações:

q. Orientações sobre os problemas de saúde?

( ) $\operatorname{sim}$ ( ) não Observações: 
4. Se a procura foi por MEDICAMENTO, e NÃO HAVIA prescrição, o paciente solicitou o nome do medicamento?

( ) $\operatorname{sim}$ ( ) não Observações:

a. Se sim, foram feitas perguntas durante a dispensação para se certificar se o medicamento seria apropriado? (Se não, ignorar as próximas 11 perguntas)

( ) $\operatorname{sim}$ ( ) não Observações:

b. Foi verificado se o paciente ou cuidador conhece o medicamento?

( ) sim ( ) não Observações:

c. Foi avaliado o motivo do uso do medicamento?

( ) sim ( ) não Observações:

d. Se foi considerado apropriado o medicamento, foi orientado o uso?

( ) $\operatorname{sim}$ ( ) não Observações:

e. Efeito esperado?

( ) sim ( ) não Observações:

f. Duração do tratamento?

( ) $\operatorname{sim}$ ( ) não Observações:

g. Interações?

( ) $\operatorname{sim}$ ( ) não Observações:

h. Reações Adversas?

( ) $\operatorname{sim}$ ( ) não Observações:

i. Se foi considerado INAPROPRIADO, foi orientado ao paciente os motivos?

( ) $\operatorname{sim}$ ( ) não Observações:

j. Foi orientado a procura por serviço médico ou outro serviço de saúde?

( ) $\operatorname{sim}$ ( ) não Observações:

k. Houve aceitação do paciente?

( ) $\operatorname{sim}$ ( ) não Observações:

I. Se não, o paciente levou o medicamento mesmo após a orientação que tal medicamento seria inapropriado?

( ) $\operatorname{sim}$ ( ) não Observações:

5. Se a procura foi por MEDICAMENTO, e NÃO HAVIA prescrição, o paciente solicitou a INDICAÇÃO de algum medicamento para tratar algum sintoma?

( ) $\operatorname{sim}$ ( ) não Observações:

a. Foram realizadas perguntas para verificar se o paciente apresentava um transtorno menor de saúde, para então escolher a melhor opção de terapia? (Se não, ignorar as próximas 24 perguntas)

( ) $\operatorname{sim}$ ( ) não Observações:

b. Foi identificado se o paciente tinha mais de 2 anos ou menos de 65 anos?

( ) $\operatorname{sim}$ ( ) não Observações:

c. Se o paciente não estava dentro dessa faixa de idade, foi orientado a procura por um atendimento médico? 
( ) $\operatorname{sim}$ ( ) não Observações:

d. Se não, o medicamento foi indicado mesmo assim?

( ) $\operatorname{sim}$ ( ) não Observações:

e. Foi perguntado sobre os sinais e sintomas do problema de saúde do paciente?

( ) $\operatorname{sim}$ ( ) não Observações:

f. Se identificado um grau de severidade dos sintomas, foi orientado a procura por um atendimento médico?

( ) $\operatorname{sim}$ ( ) não Observações:

g. Se não, o medicamento foi indicado mesmo assim?

( ) $\operatorname{sim}$ ( ) não Observações:

h. Foi verificado se os sintomas ocorrem há mais de 7 dias?

( ) $\operatorname{sim}$ ( ) não Observações:

i. Se ocorrem há mais de 7 dias, foi orientado a procura por um atendimento médico?

( ) $\operatorname{sim}$ ( ) não Observações:

j. Se não, o medicamento foi indicado mesmo assim?

( ) $\operatorname{sim}$ ( ) não Observações:

k. Foi perguntado ao paciente se o mesmo já iniciou algum tratamento para tratar esses sintomas?

( ) $\operatorname{sim}$ ( ) não Observações:

I. Se já iniciado, houve melhora?

( ) $\operatorname{sim}$ ( ) não Observações:

m. Foi perguntado ao paciente se o mesmo apresenta outros problemas de saúde?

( ) $\operatorname{sim}$ ( ) não Observações:

n. Foi perguntado ao paciente se o mesmo usa outros medicamentos?

( ) $\operatorname{sim}$ ( ) não Observações:

o. Foi verificado se o paciente tem outras situações especiais como: gravidez, lactação, alergias...?

( ) $\operatorname{sim}$ ( ) não Observações:

p. Foi identificado ser um transtorno menor de saúde? Se sim, qual?

( ) $\operatorname{sim}$ ( ) não Observações:

q. Foi indicado algum MIP? Qual?

( ) $\operatorname{sim}$ ( ) não Observações:

r. Foi indicado algum medicamento tarjado? Qual?

( ) $\operatorname{sim}$ ( ) não Observações:

s. Foi indicado algum tratamento não farmacológico? Qual?

( ) $\operatorname{sim}$ ( ) não Observações:

t. Foi indicado algum tratamento fitoterápico? Qual?

( ) $\operatorname{sim}$ ( ) não Observações:

u. Após a escolha do tratamento, o medicamento foi dispensado orientando o uso? 
( ) sim ( ) não Observações:

v. Efeito esperado?

( ) $\operatorname{sim}$ ( ) não Observações:

w. Duração do tratamento?

( ) $\operatorname{sim}$ ( ) não Observações:

x. Interações?

( ) sim ( ) não Observações:

y. Reações Adversas?

( ) $\operatorname{sim}$ ( ) não Observações: 